\title{
Experimental Determination of the Speed of Sound in Viscoelastic Pipes
}

\author{
Matjaz Prek \\ University of Ljubljana, Faculty of Mechanical Engineering, Askerceva 6, 1000 Ljubljana, Slovenia
}

(Received 31 January 2000; revised 19 April 2000; accepted 28 April 2000)

\begin{abstract}
Vibro-acoustic energy travels not only as sound waves through a fluid medium, but also as longitudinal and flexural waves through the pipe walls in a hydraulic system. Longitudinal waves in the pipe wall are coupled to the sound waves inside according to Poisson's ratio. One of the most common examples of this form of interaction is observed in water-filled pipes in which the flexibility of the walls significantly alters the speed of propagation of acoustic disturbances along the pipe. This interaction is most evident in viscoelastic pipes, which takes into account the effect of the viscoelasticity of the pipe wall material on the wave propagation. In order to determine the speed of sound in water-filled pipes, the pressure transfer function between points along the pipe is experimentally determined. Pressure transducers (hydrophones) are used to measure the fluid-borne sound pressure. The equivalent wavespeed and frequency-dependent attenuation factor is determined from this measured data. With this data, the standard impedance or transfer matrix can be derived directly to analyse the resonance conditions in hydraulic lines. Experimental results for four different pipe wall materials, with particular applications in water supply installations, are presented.
\end{abstract}

\section{INTRODUCTION}

Although it is well known that the nonlinearity of pipe wall materials and the dynamic effects of pipe walls may result in a frequency-dependent wavespeed, there has not been much research in this field. Fanelli ${ }^{1}$ has shown theoretically that the dynamic effect of the rock mass surrounding a rockbored penstock led to a complex-valued and frequencydependent Young's modulus for the wavespeed, which propagates the dispersive wave. Similarly, Fanelli et al. ${ }^{2}$ have reported on an experimental method applied to a laboratory perspex pipeline. The wavespeed in the pipeline was frequency-dependent and complex valued as a consequence of the hysteretic and dynamic properties of perspex. Franke and Seyler adopted a frequency-dependent damping factor to compute hydraulic transients in viscoelastic pipes. ${ }^{3}$ They used the impulse response method instead of the standard method of characteristics (MOC). In the MOC, a term representing the retarded wall deformation is included and evaluated using the creep compliance of the wall material by means of Kelvin-Voigt elements. Rieutord and Blanchard adapted the standard MOC and presented a theoretical study of the effect of the viscoelastic properties of the wall material on transients. ${ }^{4}$ Gally et al. compared the calculated waterhammer in polyethylene pipes with laboratory test data. ${ }^{5}$ Gl ney have proposed a modified MOC model which takes into account the effects of time-varying diameter and thickness and frequency-dependent friction. ${ }^{6}$ Ghilardi and Paoletti have investigated the application of viscoelastic pipes added to pipeline systems as suppressers of pressure surges. ${ }^{7}$

In methods based on frequency response, the effect of viscoelastic properties is modelled through a frequencydependent wavespeed and a separate frequency-dependent damping factor. Meissner has derived the wavespeed and damping factor for an oscillating pressure wave propagating in a thin-walled viscoelastic pipe by incorporating the com- plex creep compliance into the unsteady momentum and continuity equations. ${ }^{8}$ Hirschmann has studied the resonating conditions in viscoelastic pipes with a modified impedance method, using frequency-dependent wavespeed and a damping factor. ${ }^{9}$ The impulse response method has been utilised by Franke and Syler to calculate the waterhammer. ${ }^{3}$ Similarly, an impulse response method applied to compute nonperiodic transients has been proposed by other authors, e. g. Suo and Wylie. ${ }^{10,11}$ The complex wavespeed (complex-valued and frequency-dependent) is used in the standard impedance or transfer matrix method to analyse the oscillatory flow. A similar method has been proposed by Munjal and Thawani who applied the concept of transmission loss instead of the concept of wall impedance. ${ }^{12}$ In almost all studies, the pipe wall has been assumed to be of infinite length and, therefore, the effect of pipe end support conditions on the wave propagation characteristics of the pipe was not included. With regard to the coupled vibrations of the pipe and the fluid in the pipe, Wiggert et al. have considered the effect of the pipe elbow restraint on the pressure transients in the elbow. ${ }^{13}$ Lesmez et al. have presented a study on the Poisson-coupled vibrations in an elastic pipe and the fluid in the pipe which yielded transfer matrix equations relating the amplitude quantities of motion transmitted through a length of pipe. ${ }^{14}$ The amplitude relationship is good enough for modal analysis and useful for deriving the transmission loss matrix of a pipe, but is not suitable for studying the complete transmission of the sound pressure wave (amplitude and phase) through a viscoelastic pipe. An extended method, which uses the static mechanical properties and frequency-dependent mechanical properties of the pipe wall, has been proposed by Yu and Kojima. ${ }^{15}$

In order to determine the sound speed, a transfer function method is proposed. Compared to the transfer matrix method, the transfer function method is based on an analysis of the transfer function relationships between three sound pressure measurements in a pipe. The effect of the viscoelasticity of 